\title{
Analysis of Ball Screw Pair Jamming Mechanism in EMAs
}

\author{
HUILI DONG 1, YUANXUN FAN², MINGHUI LIU2', ZHIYUAN YU1
}

1 Beijing Institute of Precision Electromechanical Control Equipment

2 Nanjing University of Science and Technology

\begin{abstract}
Electromechanical actuators (EMAs) are gradually becoming a popular alternative to traditional hydraulic actuators due to w eight reduction, improved maintenance, and more feasible to realize integrated control for flight control executive system. Regarding the utilization of EMAs in the primary control surface, the major concern is the mechanical jamming, which is critical and potentially disastrous for aircraft. Therefore, the jamming mechanism and jam-tolerant capability of the ball screw transmission widely used in EMAs are researched in this paper to find the fact that causes jam failure. Dynamic analysis of the ball in raceway and passing through the returner is conducted considering elastic-plastic deformation, sliding friction, lubricant viscous resistance and impact effects to illustrate the potential correlation between the jam mechanism and operating performance. The friction self-locking critical condition for the ball in the racew ay is derived, which could be seen as a criterion for the occurrence of the jam. The variation of contact angle and friction coefficient in different operating conditions of load, velocity and frequency are analyzed and compared. The structure parameters as altitude intercept between the racew ay and returner, the angle between the return curve and the spiral line are also discussed.
\end{abstract}

\section{Introduction}

With the development of the more electric aircraft (MEA) electromechanical actuator (EMA) are gradually becoming a popular alternative to traditional hydraulic actuators. The electromechanical actuator (EMA) brings weight reduction, improved maintenance and becomes more feasible to realize integrated control for flight control executive system. However, regarding the utilization of EMAs in the primary control surface, the major concern is the mechanical jamming which is critical and potentially disastrous for aircraft. The mechanical jamming mainly refers to the ball screw pair which is widely used in EMAs.

In terms of the research on jamming mechanism, a consensus has not yet been reached. Due to the particularity of various loads and w orking conditions, the bearing characteristics, failure forms and jamming mechanisms are usually different. The dynamic behavior of ball screw pairs under high speed or overload are typically complicated and not systematically studied from theoretical and experimental aspects. Accordingly, the design reliability in some extent could not be ensured. Under the w orking conditions of high speed and heavy load, the collision impacts of the balls in raceway, the plastic deformation between two contact surfaces, as well as rapidly changing curvature of the return groove may cause jamming failure.

Therefore, the jamming mechanism and jam-tolerant capability of the ball screw transmission are researched and discussed in this paper to find the fact that causejam failure. Firstly, the structure composition and working principle of the ball screw pair is introduced. Then a dynamical model is established considering elastic-plastic contact deformation. The friction self-locking criterion for the ball in the racew ay is proposed, which could be seen as a criterion for the occurrence of jamming. The variation of contact angle and sliding friction coefficient $w$ ith different loads and speeds are analyzed and compared. Finally, optimized design ideas and improved operating parameters are suggested from the view of reducing the probability of jamming.

\section{Modeling}


Ball screw pair is widely used in electric mechanical actuating systems, which acts as the device to transmit rotational motion into linear motion. The ball screw pair is composed of screw, ball, nut and returner. In general, the motor drives the screw to rotate, which produces an axial driving force on nut though the spiral distributed balls in racew ay then outputs translational motion to the rudder.

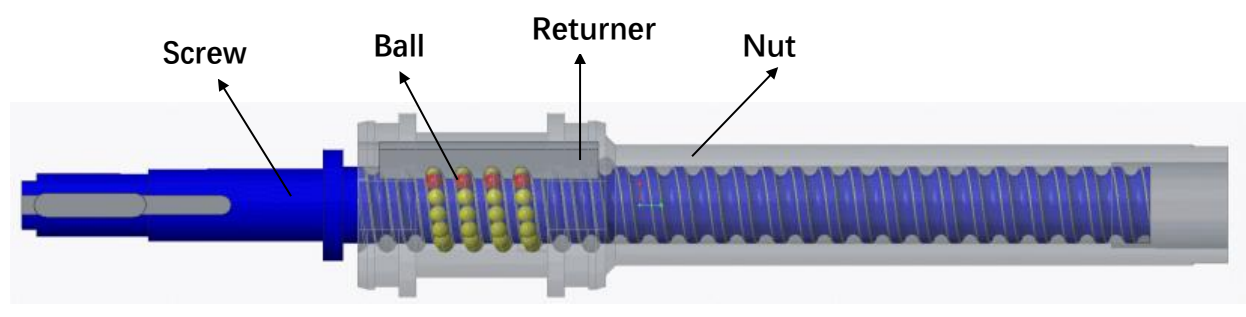

Figure 1. Ball screw pair of 3D model.

Based on the exploration of previous studies, the reasons for jamming failure of ball screw pairs could be attributed to the following situation: 1 ) friction self-locking due to plastic deformation or wear in the raceway. In the condition of overloads exceeding normal level, plastic deformation and wear accumulates gradually in the contact regions, then the contact parameters of the ball and the racew ay have changed, and finally lead to friction self-locking. 2) Fatigue damage can also lead to jam failure. The ball screw pair subjected to heavy load in frequency reciprocating motion will easily cause the ball to crack or the top tooth of the racew ay to break.3) The impact deform ation and wear of the returner cause jam failure. Under heavy load and high-speed conditions, the ball will have a strong impact when entering the raceway. The ball suffering collision force would produce large deformation and develop serious wear. 4) When the ball screw pair is in high-frequency reciprocating conditions, the stress state of the ball above the top bud of the screw racew ay are constantly changing. Due to the hysteresis of the force transmission, the ball may not be able to accommodate the su dden change of the motion state, and lead to self-locking problem.

All of performances described above may cause jam failure, so a dynamic model considering elastic-plastic deformation and impacts should be built to illustrate the potential cor relation bet ween the jam mechanism and operating performance.

\subsection{Contact Analysis}

First, the coordinate systems of the ball screw pairs is established for the analy sis of contact force, as show $n$ in Fig.2, in which $r_{m}$ and $\gamma$ is respectively the pitch radius and the helix angle, as $\omega$ is the angular velocity the screw.

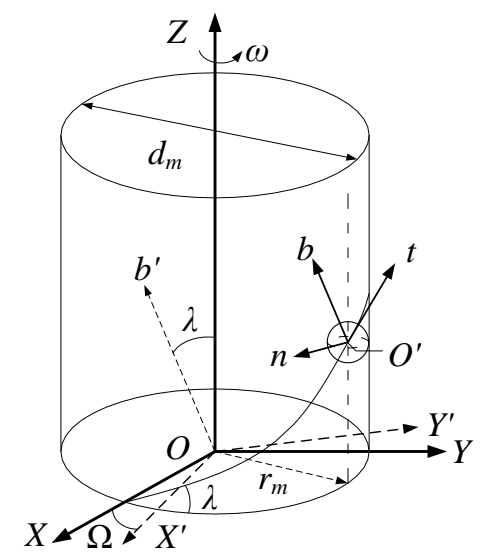

(a)The Cartesian coordinates and Frenet coordinates 


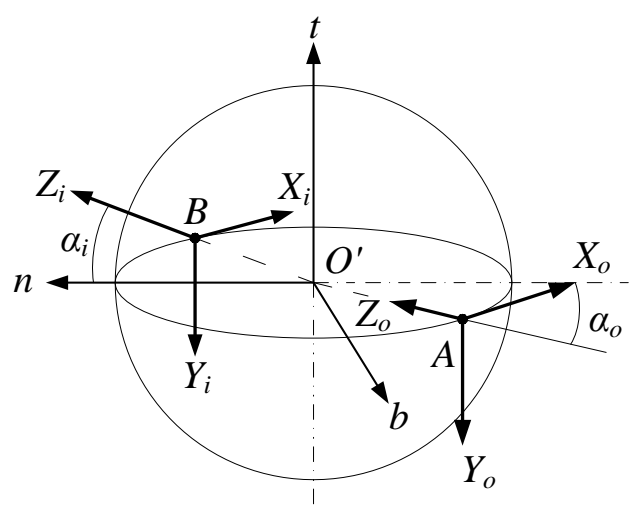

(b) The local coordinate system

Figure 2. The ball screwspace coordinate system.

Accordingly, the transformation matrix between two coordinate system $O-X Y Z$ and $O^{\prime}$-tnb is describe as

$$
\left[\begin{array}{c}
X \\
Y \\
Z
\end{array}\right]=\left[\begin{array}{ccc}
-C_{\alpha} S_{\varphi} & -C_{\alpha} & S_{\alpha} S_{\varphi} \\
C_{\alpha} C_{\varphi} & -S_{\varphi} & -S_{\alpha} S_{\varphi} \\
S_{\alpha} & 0 & C_{\alpha}
\end{array}\right]\left[\begin{array}{ccc}
\cos \Omega & -\sin \Omega & 0 \\
\sin \Omega & \cos \Omega & 0 \\
0 & 0 & 1
\end{array}\right]\left[\begin{array}{c}
t \\
n \\
b
\end{array}\right]
$$

The coordinate transformations between the local coordinates and the Frenet coordinates are given as

$$
\left[\begin{array}{c}
X_{n^{\prime}} \\
Y_{n^{\prime}} \\
Z_{n^{\prime}}
\end{array}\right]=\left[\begin{array}{ccc}
0 & -\sin \alpha_{n^{\prime}} & \cos \alpha_{n^{\prime}} \\
1 & 0 & 0 \\
0 & \cos \alpha_{n^{\prime}} & \sin \alpha_{n^{\prime}}
\end{array}\right]\left[\begin{array}{l}
t \\
n \\
b
\end{array}\right]
$$

The ball rolls in raceway with partial slide movement. The slip angle and sliding velocity is defined in Fig.3, which could be written as ${ }^{[1]}$

$$
\psi_{o}=\tan ^{-1}\left(\frac{V_{Y o}}{V_{\mathrm{Xo}}}\right)+\pi
$$

The relationship betw een $\mathrm{V}_{\mathrm{y}_{0}}$ and $\mathrm{V} \mathrm{x}_{\mathrm{o}}$ could be calculated by

$$
\frac{V_{Y o}}{V_{X o}}=\frac{d \omega_{m}+r_{b}\left(\omega_{b} \cos \alpha_{o}-\omega_{n} \sin \alpha_{o}\right)}{-r_{b} \omega_{t}}
$$

Accordingly, the slip angle and slide ratio at the contact point could be expressed as

$$
\begin{gathered}
\psi_{i}=\tan ^{-1}\left(\frac{V_{Y i}}{V_{X i}}\right)+\pi \\
\frac{V_{Y i}}{V_{X i}}=\frac{d\left(\omega_{m}-\omega\right)-r_{b}\left[\left(\omega_{b}-\omega \cos \lambda\right) \cos \alpha_{i}-\omega_{n} \sin \alpha_{o}\right]}{r_{b}\left(\omega_{t}-\omega \sin \lambda\right)}
\end{gathered}
$$




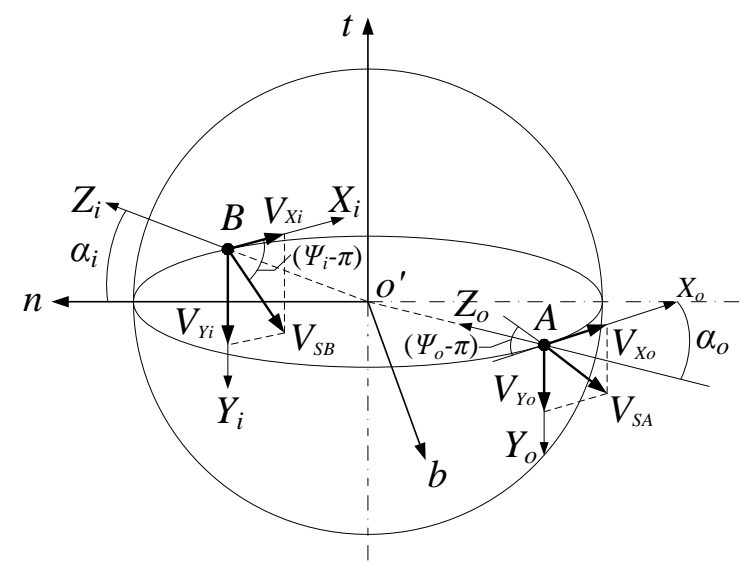

Figure 3. slip angle and sliding velocity.

Based on Jones' racew ay control theory, assuming that the curvature center of the nut raceway is fixed and the ball is solid, while the screw raceway could be deformed under external load, the contact angle between the ball and screw racew ay becomes different with the one between the ball and the nut. In that situation, the curvature center of the screw raceway will deviate from the nut, which is coincident with each other before loading.

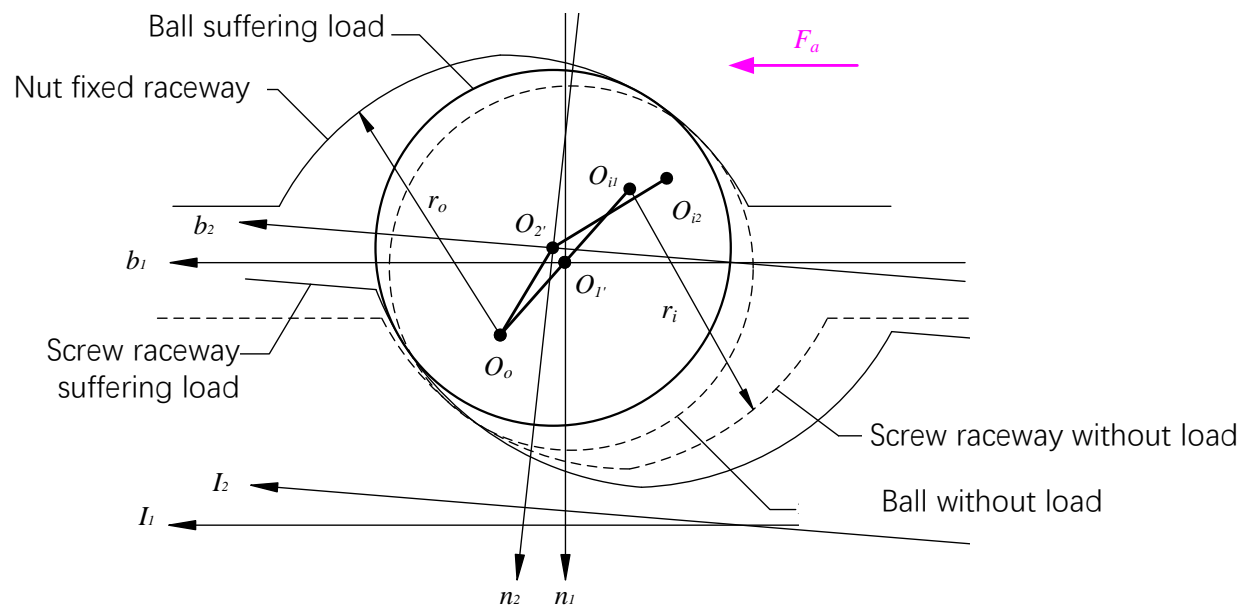

Figure 4. Change of contact position before and after loa ding.

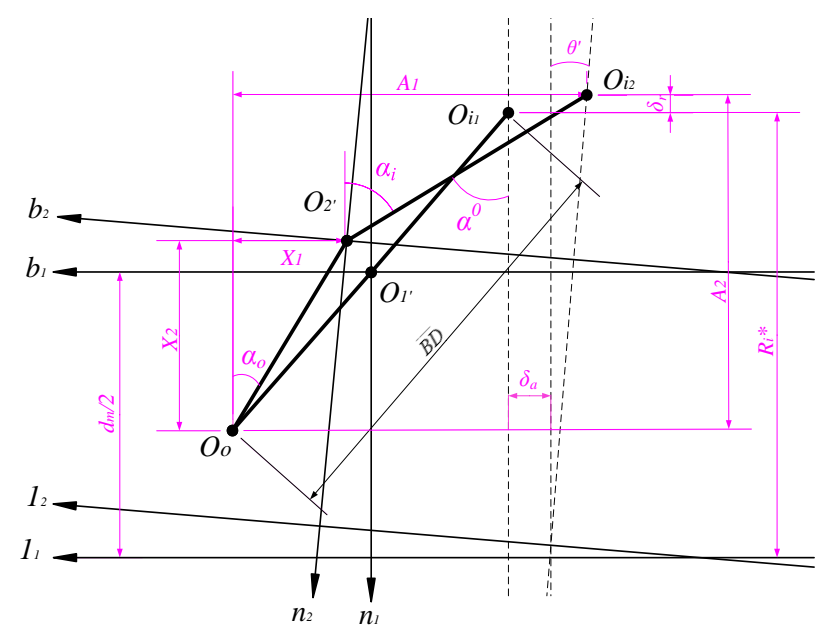

Figure 5. Center of curvature before and after loa ding. 
Fig.5 show s the moving curvature center of the ball and the racew ay at the contact point before and after loading. According to geometric equation, the deformation parameter could be calculated by following expressions:

$$
\begin{gathered}
\left\{\begin{array}{l}
A_{1}=\overline{B D} \sin \alpha^{0}+\delta_{a}+\theta^{\prime} R_{i}^{*} \\
A_{2}=\overline{B D} \cos \alpha^{0}+\delta_{r}
\end{array}\right. \\
\left\{\begin{array}{l}
\left(A_{1}-X_{1}\right)^{2}+\left(A_{2}-X_{2}\right)^{2}-\left[\left(f_{i}-0.5\right) D_{b}+\delta_{i}\right]^{2}=0 \\
X_{1}^{2}+X_{2}^{2}-\left[\left(f_{o}-0.5\right) D_{b}+\delta_{o}\right]^{2}=0
\end{array}\right.
\end{gathered}
$$

The contact angle could be obtained by

$$
\left\{\begin{array}{l}
\cos \alpha_{o}=\frac{X_{2}}{\left(f_{o}-0.5\right) D_{b}+\delta_{o}}, \sin \alpha_{o}=\frac{X_{1}}{\left(f_{o}-0.5\right) D_{b}+\delta_{o}} \\
\cos \alpha_{i}=\frac{A_{2}-X_{2}}{\left(f_{i}-0.5\right) D_{b}+\delta_{i}}, \sin \alpha_{i}=\frac{A_{1}-X_{1}}{\left(f_{i}-0.5\right) D_{b}+\delta_{i}}
\end{array}\right.
$$

Ty pically, the contact deformation should be calculated first through static analysis to obtain the contact angle. The plastic deformation can be obtained by

$$
\delta_{s}=K_{s} \cdot Q^{2}
$$

in which $\mathrm{Q}$ is normal pressure, $\mathrm{K}_{\mathrm{s}}$ is given as

$$
K_{s}=1.3 \times 10^{-7}\left(\rho_{11}+\rho_{21}\right)\left(\rho_{12}+\rho_{22}\right) / d_{b}
$$

The contact form between the ball and the raceway changes from four-point contact to two- point contact under the combined action of axial force and preload. Therefore, the force balance equation could be written as

$$
F_{a}-\sum_{j=1}^{Z} P_{j} \sin \alpha_{j} \cos \lambda=0
$$

Based on Hertz theory, the normal pressure is calculated by following equations

$$
Q_{n^{\prime}}=k_{n^{\prime}} \delta_{n^{\prime}}
$$

in which $k_{n \prime}$ ' denotes load-displacement coefficient, $\delta_{n}^{*}$ is the dimensionless contact deformation

$$
\left\{\begin{array}{l}
k_{n^{\prime}}=2.15 \times 10^{5}\left(\sum \rho_{n^{\prime}}\right)^{-0.5}\left(\delta_{n^{\prime}}^{*}\right)^{-1.5} \\
\delta_{n^{\prime}}^{*}=\frac{2 \mathfrak{J}_{n^{\prime}}}{\pi}\left(\frac{\pi}{2 \bar{K}_{n^{\prime}}^{2} \wp_{n^{\prime}}}\right)^{1 / 3}
\end{array}\right.
$$




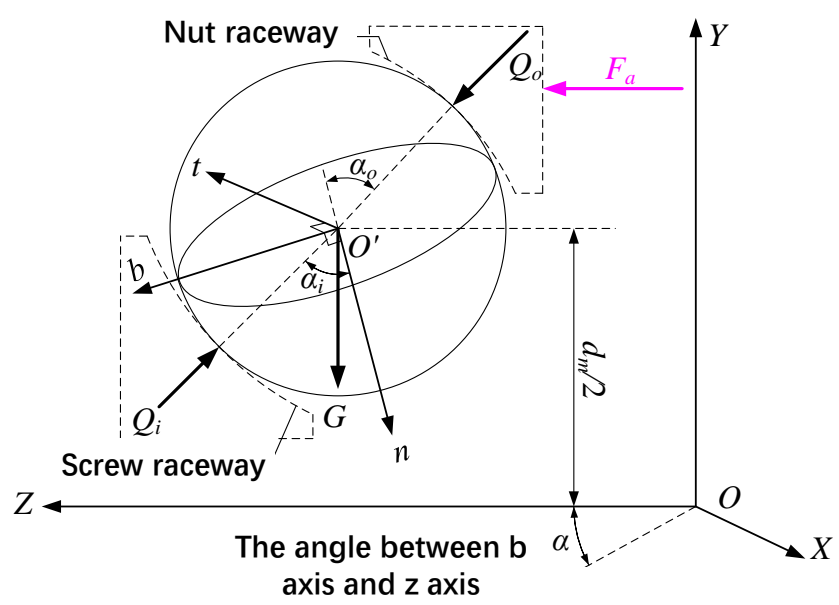

Fig.6 Contact force in static state

\section{2. dynamic model}

Taking the ball moving along the screw racew ay as analysis object, all the forces acting on the ball include sliding friction force in contact regions, lubricant viscous resistance, inertia force due to accelerating and gravity that could be ignored in some extent.

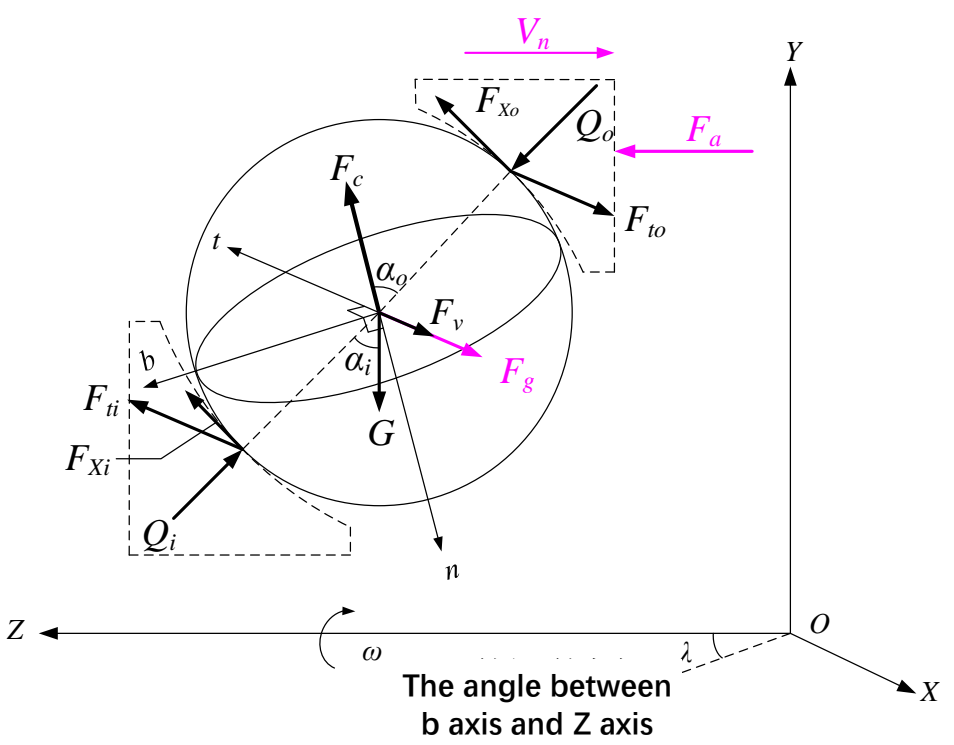

Figure 7. Force analysis of the ball suffering axialload.

When the axial external load has the same direction with the translating velocity of the nut, the forces acting on the ball in $\mathrm{t}-\mathrm{n}$ plane and b-n plane are illustrated in Fig.8. 


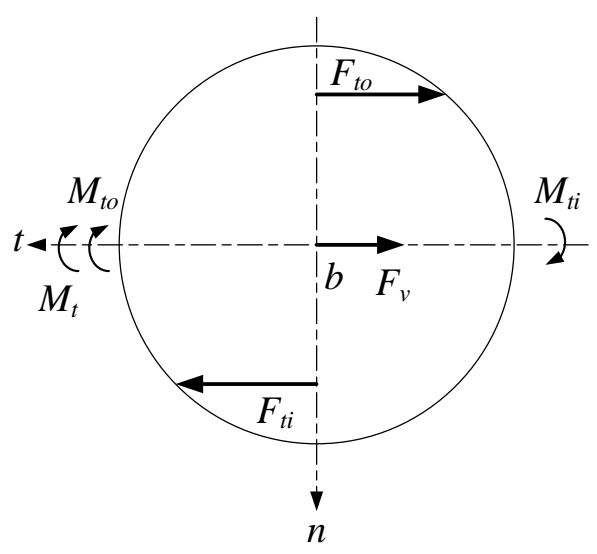

Tangent plane $b-n$

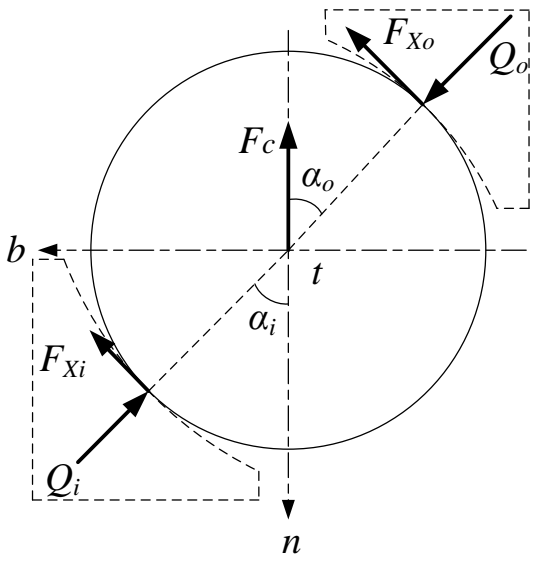

Normal plane $t-n$

Figure 8. Force in tangent plane b-n and normal plane $t-n$.

Force and moment equations in Frenet-Serret coordinates system could be written as

$$
\begin{gathered}
\text { t-direction: } F_{t i}-F_{t o}-F_{v}=0 \\
\text { t-direction: } M_{t i}+M_{t o}-M_{t}=0 \\
\text { n-direction: }-Q_{i} \cos \alpha_{i}-F_{X i} \sin \alpha_{i}+Q_{o} \cos \alpha_{o}-F_{X o} \sin \alpha_{o}-F_{c}=0 \\
\text { n-direction: } Q_{o} \sin \alpha_{o}+F_{X o} \cos \alpha_{o}-Q_{i} \sin \alpha_{i}+F_{X i} \cos \alpha_{i}=0
\end{gathered}
$$

in which $F_{c}$ indicates the centrifugal force of a single ball, $w$ hile $F_{v}$ denotes the viscous damping force, which can be obtained by ${ }^{[2]}$

$$
\begin{aligned}
F_{c}= & m r_{m} \omega_{m}^{2} \\
& F_{v}=\frac{c_{v} \pi \xi d_{b}^{2}\left(d_{m} \omega_{m}\right)^{1.95}}{32 g}
\end{aligned}
$$

The friction moment $M_{t n}$, could be calculated by ${ }^{[1]}$

$$
M_{t n^{\prime}}=a_{n^{\prime}} b_{n^{\prime}} \int_{-1}^{1} \int_{-\sqrt{1-q^{\prime 2}}}^{\sqrt{1-q^{\prime 2}}} \tau_{x n^{\prime}} r b d q^{\prime} d t^{\prime}
$$

When the direction of the axial external load is different from the translating velocity of the nut, the analysis method is similar to above expressions.

Accordingly, the friction self-locking phenomenon would occur when the contact deformation changes the contact angel and leads to a result that the sum force of friction and centrifugal force becomes bigger than the normal force in plane $t-n$.

The friction self-locking criterion could be written as

$$
\begin{gathered}
\left(F_{X o}+F_{X i}\right) \cos \left(\frac{\alpha_{i}-\alpha_{o}}{2}\right)+F_{c} \sin \left(\frac{\alpha_{i}+\alpha_{o}}{2}\right) \geq\left(Q_{o}+Q_{i}\right) \sin \left(\frac{\alpha_{i}-\alpha_{o}}{2}\right) \\
F_{X i}=Q_{i} \mu_{i} \cos \psi_{i} \\
F_{X o}=Q_{o} \mu_{o} \cos \psi_{o}
\end{gathered}
$$

When the axial external load has the same direction as the translating velocity of the nut, the translating velocity of the nut, the friction coefficient $\mu \mathrm{i}$ equals to $\mu \mathrm{o}$, and can be both written as $\mu$. Therefore, the friction coefficient should be satisfy the following relationship 


$$
\mu \geq \sin \psi \tan \left(\frac{\alpha_{i}-\alpha_{o}}{2}\right)
$$

To simplify above expression, assume that $\sin \psi \approx 1$, then equation (16) could be expressed as

which can also be written as

$$
\mu>\frac{\sin \alpha_{i} \cos \alpha_{o}-\cos \alpha_{i} \sin \alpha_{o}}{1+\cos \alpha_{i} \cos \alpha_{o}+\sin \alpha_{i} \sin \alpha_{o}}
$$

$$
\mu>\sqrt{\frac{-A_{1}^{2}-A_{2}^{2}+\left(\delta_{i}+\delta_{o}\right)^{2}+2 \delta_{i} d_{o}-2 \delta_{i} d_{b}+2 \delta_{o} d_{o}+\left(d_{o}-d_{b}\right)^{2}}{A_{1}^{2}+A_{2}^{2}+\left(\delta_{i}-\delta_{o}\right)^{2}}}
$$

In which $A_{1}$ and $A_{2}$ can be obtained by equation (7), $d_{0}$ is the nominal diameter of the ball screw, and $\mathrm{db}$ is the diameter of the ball.

\subsection{Force analysis of the ball in returner channel}

The ball in the returner may be stuck due to the sudden change of the w orking conditions caused by the collision and extrusion betw een the adjacent balls and the returner. Therefore, it is necessary to conduct a dynamic analysis of the balls in the returner.

Under high-frequency reciprocating conditions, the contact angle between the ball $\mathrm{O}_{2}$ at the maximum curvature position and its adjacent balls can reach the maximum value. The resultant force acting on the ball $\mathrm{O}_{2}$ will press the ball against the groove surface of the returning spiral curve of the returner, and make it difficult to turn and continue to slide forw ard.

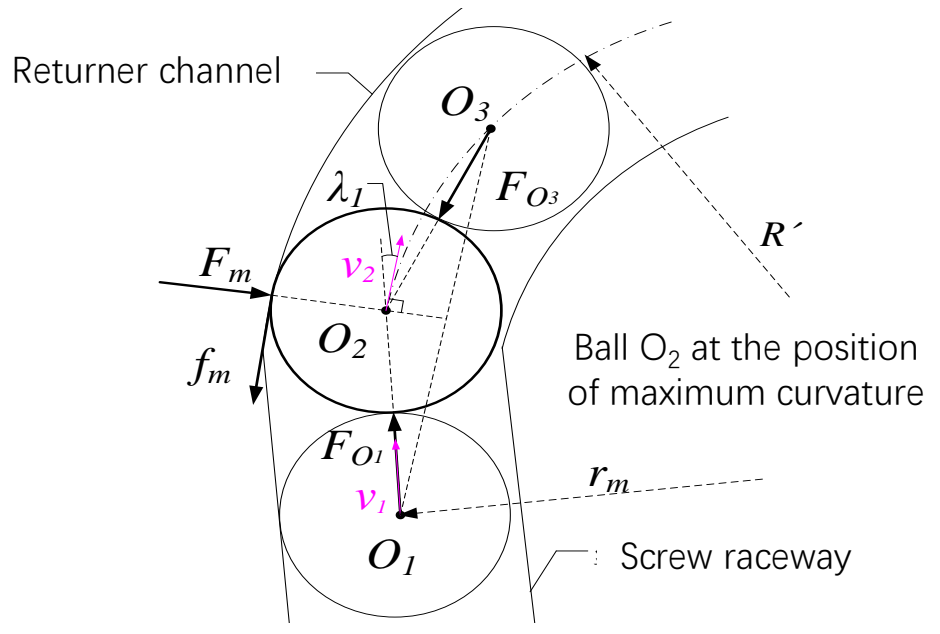

Figure 9. Forces analysis at the position of maximum curvature.

When the ball $\mathrm{O} 2$ is in a normal state of motion under the action of the thrust of the rear ball, the ball collides with theinner wall of the returner at a tangential velocity. If the ball passes through the position of maximum curvature, the thrust provided by the rear ball is not enough to make the ball in the returner continue to roll or slide against the friction force, the ball will keep in a static state, that is, a jam phenomenon occurs.

The force analysis of the ball is as follows:

$$
\left\{\begin{array}{c}
f_{m}+F_{O_{3}} \cos \lambda_{1}-F_{O_{1}} \cos \lambda_{1}=0 \\
F_{m}-\left(F_{O_{1}}+F_{O_{3}}\right) \sin \lambda_{1}=0
\end{array}\right.
$$

Based on Hertz theory, the elastic contact force $\mathrm{F}_{\mathrm{m}}$ could be w ritten as

$$
F_{m}=k_{h} \delta_{m}=\frac{1.14 m_{b} v_{2}^{2}}{\delta_{m}}
$$

When the maximum friction force in the $t$ direction generated by the contact between the ball and the screw racew ay is still less than or equal to the required thrust of the ball, the ball will stuck, or even jam. Consequently, the jam failure conditions of the ball screw pair can be obtained as: 


$$
F_{O_{1}} \geq Z F_{t i}
$$

In which $F_{01}$ is the thrust required when a single ball passes through the position of the maximum curvature of the returner; $Z$ is the effective number of balls carried by a single circle, and $\mathrm{F}_{\mathrm{ti}}$ is the sliding friction force at the contact point between the ball and the screw with the maximum force, calculated by the following equation

$$
F_{t i}=\mu_{i} Q_{i} \sin \psi_{i}
$$

According to the current machining accuracy of the corresponding holes on the return device and the nut, the diameter of the return circulation channel is usually controlled within the range of $0.3 \mathrm{~mm}$ to $0.7 \mathrm{~mm}$ greater than the ball diameter [18]. Therefore, the ball must pass a "step" in the process of entering and exiting the return channel,

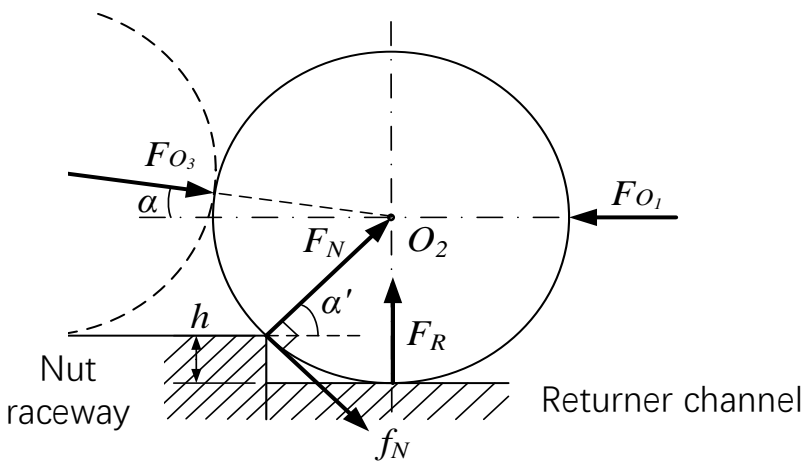

Figure 10. Force of the ball entering the nut raceway from returner.

The force and moment balance equations when the ball enters the returner are

$$
\left\{\begin{array}{l}
F_{O_{3}} \cos \alpha+F_{N} \cos \alpha^{\prime}+f_{N} \sin \alpha^{\prime}-F_{O_{1}}=0 \\
F_{N} \sin \alpha^{\prime}-f_{N} \cos \alpha^{\prime}+F_{R}-F_{O_{3}} \sin \alpha=0 \\
k_{R} F_{N}=F_{O_{1}} r_{b} \sin \alpha^{\prime}+F_{R} r_{b} \cos \alpha^{\prime}-F_{O_{3}} r_{b} \sin \left(\alpha+\alpha^{\prime}\right)
\end{array}\right.
$$

Where FR denotes the normal force between the ball and racew ay of the returner, is rolling friction fore at contact point, $f_{N}=\mu^{\prime} F_{N}$, in which $\mu^{\prime}$ is rolling friction coefficient, $k_{R}=\mu^{\prime} r_{b}, F_{01}$ and $F_{03}$ are the force applied to $\mathrm{O}_{2}$ from adjacent balls $\mathrm{O}_{1}$ and $\mathrm{O}_{3}$.

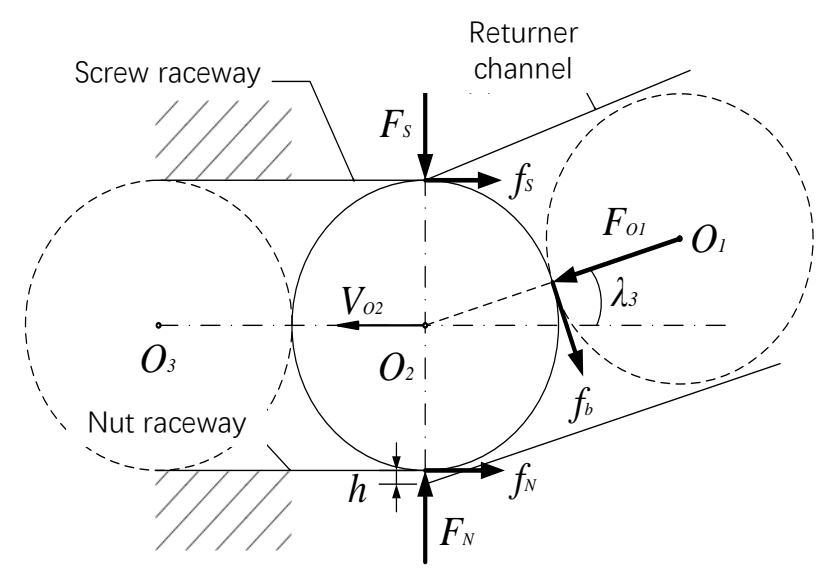

Figure 11. Forces of the ball at the position entering the screw raceway.

The force analysis of the ball $\mathrm{O}_{2}$ located directly above the crest of the screw racew ay is obtained 


$$
\left\{\begin{array}{l}
f_{S}+f_{b} \sin \lambda_{3}+f_{N}-F_{O_{1}} \cos \lambda_{3}=0 \\
F_{N}-F_{S}-f_{b} \cos \lambda_{3}-F_{O_{1}} \sin \lambda_{3}=0 \\
f_{S}=\mu F_{S} \\
f_{N}=\mu F_{N} \\
f_{b}=\mu F_{O_{1}}
\end{array}\right.
$$

Simplified as

$$
F_{O_{1}}=\frac{2 F_{N}}{\left(\mu+\frac{1}{\mu}\right) \cos \lambda_{3}}
$$

\section{Numerical solution}

The flow chart of the numerical solution is shown in Fig.12. Firstly, the axial deformation and the radius deformation are calculated by equation (7) based on the Jones' racew ay theory to obtain the initial contact angle. Then the angular velocity of the ball's revolution and spinning as well as the slip angle is calculated. Then the friction coefficients are solved to update the contact deformation and the slip angle until the convergence criterion is satisfied. Based on the results of friction coefficients the self-locking condition is given to assess whether jam occurs. Table 1 gives the ball screw pair parameters for numerical solution.

\begin{tabular}{|c|c|c|c|c|c|}
\hline \multicolumn{2}{|c|}{ Ball diameter $\mathrm{d}_{\mathrm{b}}$} & $3.5 \mathrm{~mm}$ & \multicolumn{2}{|c|}{ Number of balls $Z^{\prime}$} & 44 \\
\hline \multicolumn{2}{|c|}{ Curvature ratio $\mathrm{t}$} & 1.07 & \multicolumn{2}{|c|}{ Poisson's ratio $\mu$} & 0.3 \\
\hline \multicolumn{2}{|c|}{ Initial contact angle } & $45^{\circ}$ & \multicolumn{2}{|c|}{ Helix angle $\lambda$} & $6.807^{\circ}$ \\
\hline \multirow{2}{*}{$\begin{array}{l}\text { Material } \\
\text { properties }\end{array}$} & Nut/screw & $\sigma_{\mathrm{s}}=1152 \mathrm{MPa}$ & \multirow{2}{*}{$\begin{array}{l}\text { Elastic } \\
\text { Modulus } \\
\text { E }\end{array}$} & Nut/screw & $2 \times 10^{5} \mathrm{MPa}$ \\
\hline & ball & $\sigma_{\mathrm{s}}=1617 \mathrm{MPa}$ & & ball & $2.07 \times 10^{5} \mathrm{MPa}$ \\
\hline
\end{tabular}

Table 1. Ball screw pair parameters list. 


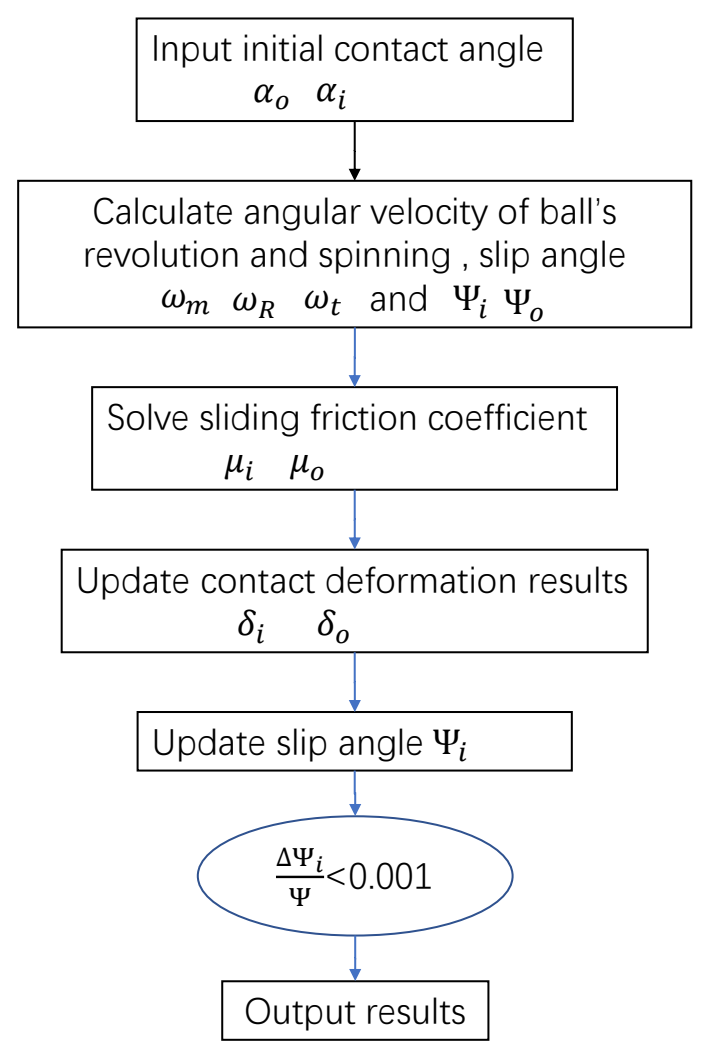

Figure 12. Flow chart of numerical solution.

\section{Results}

In order to reveal the jam mechanism of the ball screw, this paper discusses the influence of different axial forces and speeds on the contact angle, sliding friction coefficient, as w ell as the change law of viscous damping force and centrifugal force. Then, the kinematics of the ball with different frequencies is discussed.

Fig.13 shows the changing curve of the contact deformation between the ball and the raceway with the increasing speed and axial force within $8 \mathrm{KN} \sim 24 \mathrm{KN}$. The contact deformation at this time is the sum of the elastic deformation and the plastic deformation. It can be seen that as the axial force increases, a certain amount of plastic deformation occurs at the contact region, and the total amount of contact deformation keeps increasing with the axial force.

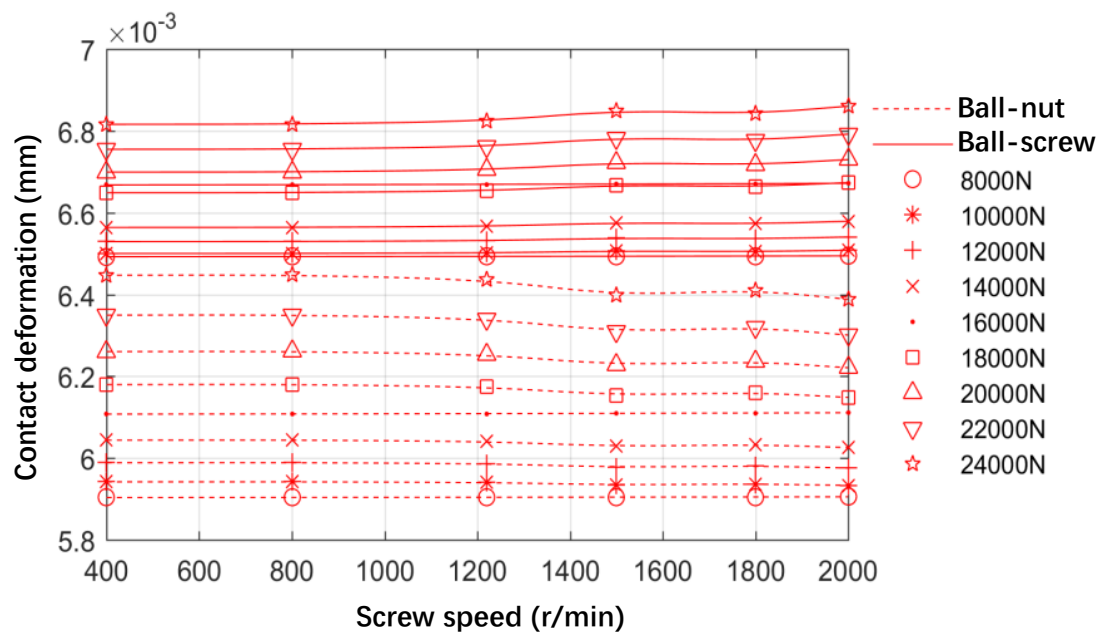

Figure 13. Contact deformation with increasing speed and axial force. 
Fig.14 show s the curve diagram of the contact angle between the ball and the raceway under different working conditions.

As shown in Figure1, the contact angle $\alpha_{\mathrm{i}}$ increases with increasing speed, but decreases with the increase of axial force, while $\alpha_{\mathrm{o}}$ has opposite trend with speed and axial force . When the axial force $\mathrm{Fa}<16 \mathrm{kN}$, the contact angle $\alpha_{\mathrm{i}}$ is higher than $\alpha_{\mathrm{o}}$. However, as the axial force increases, the plastic deformation has an increased proportion and the difference between the two contact angles has been shrinking, until $\alpha_{i} \approx \alpha_{o}$ where Fa reaches $24 \mathrm{kN}$.

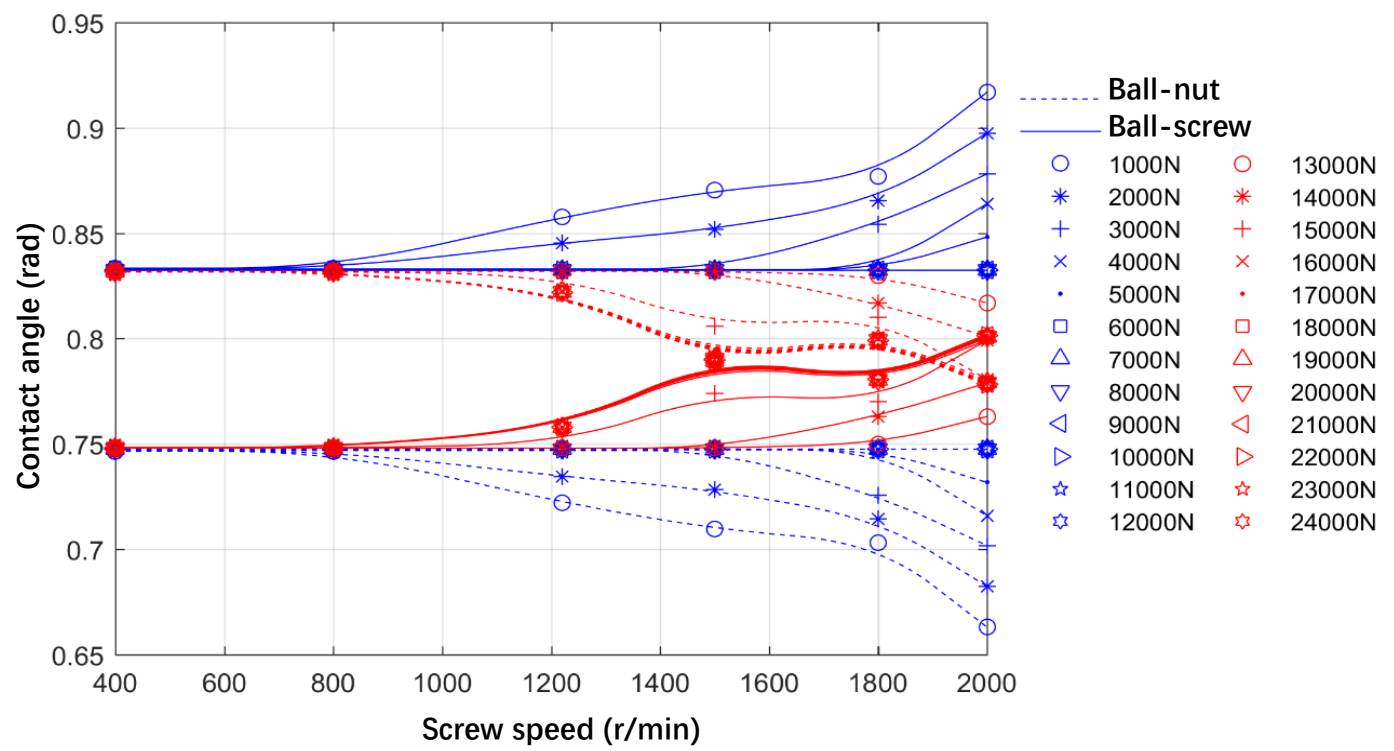

Figure 14. variation of contact angle between ball and raceway.

Fig.15 is the curve of the sliding friction coefficient between the ball and the raceway with increasing speed and axial force. As can be seen from the figure, since the increase in plastic deformation is extremely small compared to the maximum elastic deformation, the sliding friction coefficient remains basically unchanged when rotational speed keeps in a constant value. Generally, the sliding friction coefficient will increase as the speed becomes high.

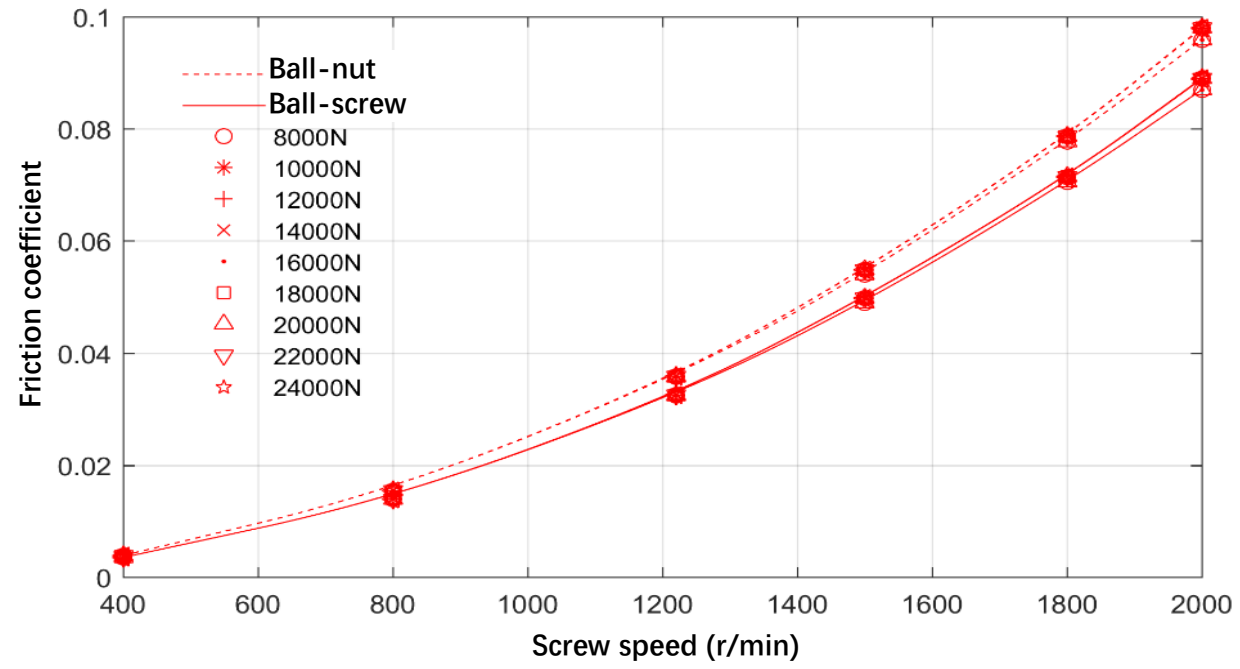

Figure 15. Va riation of friction coefficient with incre a sing speed and axial force.

Due to the influence of centrifugal force, the friction coefficient of the contact between the ball and the nut raceway is greater than that of the contact with the screw raceway. Within the range of the initial yield load, both the axial force and the speed have a great influence on the sliding friction coefficient. The sliding friction coefficient decreases with the increase of the axial force, and increases with the increasing speed. When the axial force is greater than the initial yield load, the rotation speed 
becomes a key factor affecting the sliding friction coefficient, which increases with the increase of the rotation speed.

The revolution and rotation angular velocity of the ball are shown in Figure4. It can be found that the rotation angular velocity of the ball is approximately 5 times the revolution angular velocity. From equations (3.30) and (3.34), it can be seen that the centrifugal force Fc and the lubricant viscosity resistance $\mathrm{Fv}$ are only related to the revolution angular velocity $\omega \_\mathrm{m}$ of the ball. Therefore, the centrifugal force and lubricant viscosity resistance of the ball at different speeds are shown in Figure 4.

From Fig.17, the viscous resistance and centrifugal force of the lubricant that the ball receives increases with the increase in speed, but the value is very small, which has little effect on the sliding movement of the ball. Therefore, the centrifugal force could be ignored when deriving self-locking condition.

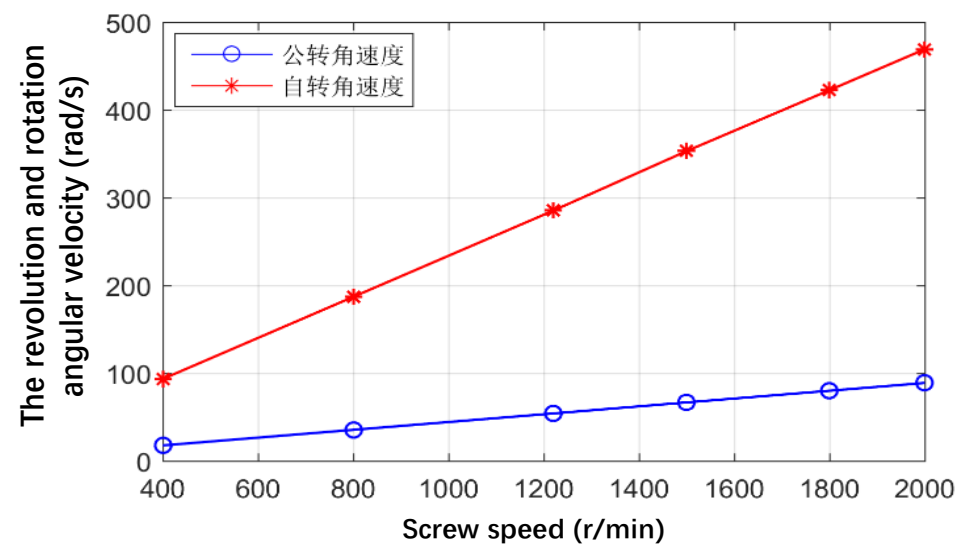

Figure 16. The revolution and rotation angular velocity of the ball.

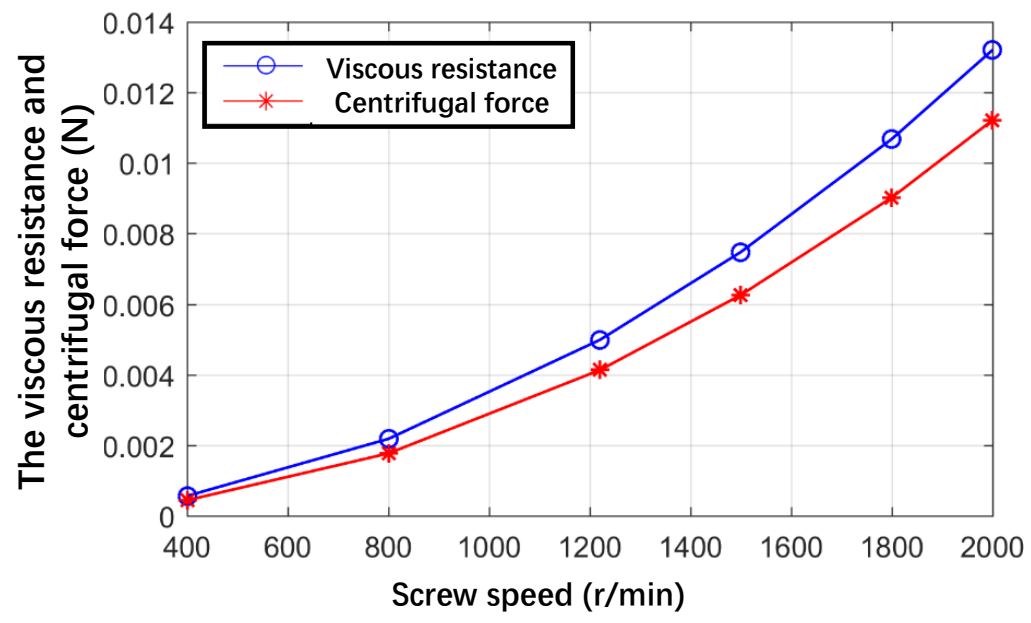

Figure 17. The viscous resistance and centrifugal force with incre asing speed.

Fig.18 is the theoretical values of the thrust and friction force under different w orking conditions. It can be inferred that when the axial force is constant, the greater the speed, the more difficult it is for the sum of the frictional force in the tangential direction of the single-turn ball helix to meet the thrust required by the ball passing through the returner, so it is easier to occur jam and accelerates the failure of the ball screw pair. 


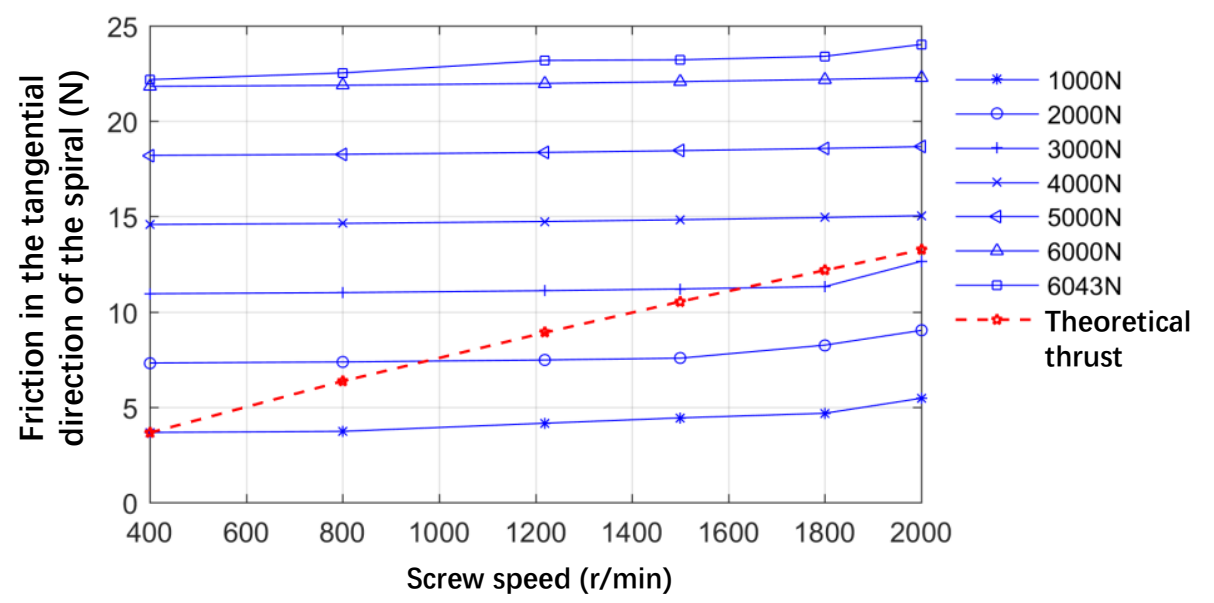

Figure 18. Thrust required and friction force under diffe rent axial forces and speeds.

Fig.19 is the curve of the required thrust with the angle $\lambda_{3}$ between the return curve and the spiral. It can be found that when the axial force, pre-tightening force and working conditions remain unchanged, the greater the angle between the return curve and the helix, the greater the thrust required.

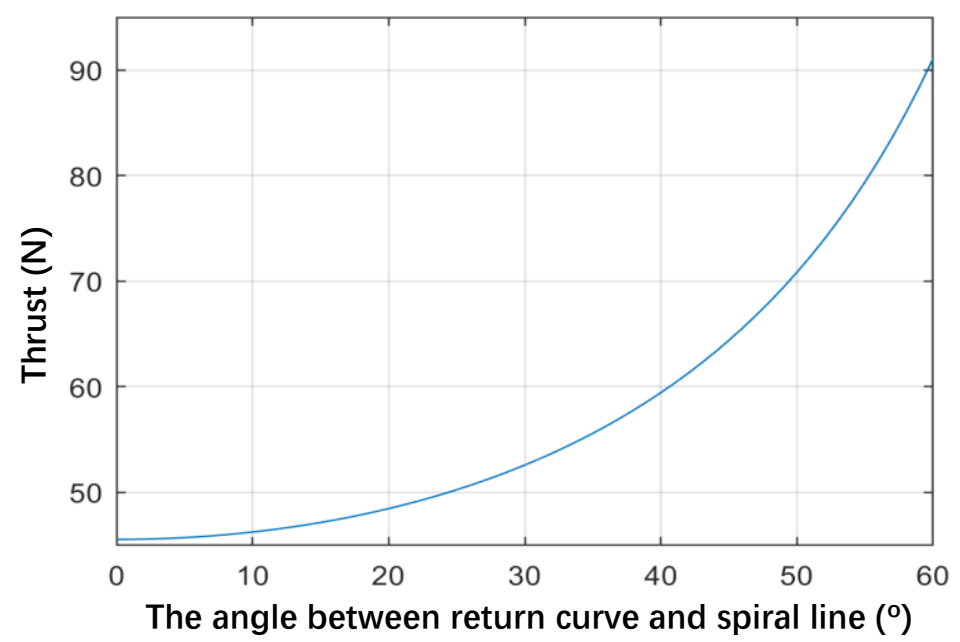

Figure 19. The required thrust with increa sing angle between return curve and spiral line .

It can be seen from the above analysis that the height $h$ and the angle between the ball return curve and the spiral line have a great influence on the required thrust of the ball. The greater the height and the included angle, the greater the thrust required for the ball to pass through the returner, which makes the frictional moment abrupt and increases the unevenness of the ball's movement. When the maximum torque that the motor can provide is exceeded, a "stuck" phenomenon will occur. Therefore, in the design of the return device, the height difference between the return channel and the nut racew ay and the angle betw een the return curve and the spiral line should be minimized to enhance the smoothness of the ball movement and prevent jamming.

\section{Conclusion}

This paper establishes a dynamic model of the ball screw pair considering the elastic-plastic contact deformation, centrifugal force, and viscous resistance to analyzes the stress state of the ball in the raceway, and discuss the influence of the axial force and speed on the contact and sliding friction coefficient to derive the conditions that cause friction self-locking. The force of the balls in and out of the returner is analyzed, and the influence of the height difference, the angle between the return curve and the spiral line on the thrust required for the balls to enter the racew ay is calculated. 
For the ball chain in the cycle, reducing the friction and collision between adj acent balls is beneficial to reduce the impact load and improve the high-speed performance of the ball screw pair.

From the perspective of the circulation structure, when designing and manufacturing the high load internal circulation ball screw pair returner, attention should be paid to improving the accuracy of the connection betw een the racew ay in the returner and the entrance and exit of the screw raceway. When designing the ball returning groove curve, the relationship between the tangent line of the ba ll rotation and the angle of rise must be handled well to make the ball move smoothly which could avoid undesirable phenomena such as jamming, and reduce impact effects.

\section{References}

[1]Wei C.C, Lin F.J. Kinematic Analysis of the Ball Screw Mechanism Considering Variable Contact Angles and Elastic Deformations. Journal of Mechanical Design, 125 (4) (2003) 717-733.

[2]Harris T. A. 1984, Rolling Bearing Analysis. John Wiley \& Sons, New York.

[3] M. Izaw a, H. Shimoda.Study on the load distribution in the ball screw, Japan Journal of Precision Machine 42 (11) (1976)1021-1028.

[4]Cheng Q., Qi B. B., Liu Z.F.. An accuracy degradation analysis of ball screw mechanism considering time-varying motion and loading w orking conditions, Mechanism and Machine Theory $134(2019) 1-23$

[5]C. C. Wei , J.F. Lin , J. Horng , Analysis of a ball screw with a preload and lubrication, Tribol. Int. 42 (2009) 11-19.

[6]C. Wei , R. Lai, Kinematical analyses and transmission efficiency of a preloaded ball screw operating at high rotational speeds, Mech. Mach. Theory 46 (7) (2011) 880-898.

[7]X.M. Kang , L. Wang, Experimental analysis of axial load internal circulation ball screw pair of friction torque and the impact, Appl. Mech. Mater. 401-403 (2013)139-145.

$[8] \mathrm{N} . \mathrm{Xu}, \mathrm{W}$. Tang, Modeling and analyzing the slipping of the ball screw, Latin Am. J. Solids Struct. 12 (3) (2015) 612-623.

[9]C. Wei , W. Liou , R. Lai , Wear analysis of the offset type preloaded ball-screw operating at high speed, Wear 292 (2012) 111-123.

[10]J.Hu , M. Wang, T. Zan, The kinematics of ball-screw mechanisms via the slide-roll ratio, Mech. Mach. Theory 79 (2014) 158-172 .

[11]Xue Song Mei. Study on the load distribution of ball screw with errors. Mechanism and Machine Theory,2003(38): 1258-1268.

(C) 2020 by the authors; licensee MDPI, Basel, Switzerland. This article is an open access article dis tribute $\mathrm{d}$ under the terms and conditions of the Creative Commons by Attribution (CC-BY) license (http://creativecommons.org/licenses/by/4.0/). 